\title{
American College of Radiology \\ Contrast Enhanced Ultrasound Liver Imaging Reporting and Data System (CEUS LI-RADS) for the diagnosis of Hepatocellular Carcinoma: a pictorial essay \\ American College of Radiology \\ Contrast Enhanced Ultrasound Liver Imaging Reporting and Data System (CEUS LI-RADS) für die Diagnose von Hepatozellulären Karzinomen: ein Bild-Essay
}

Authors

Fabio Piscaglia', Stephanie R. Wilson², Andrej Lyshchik ${ }^{3}$, David Cosgrove ${ }^{4}$, Christoph F. Dietrich ${ }^{5}$, Hyun-Jung Jang ${ }^{6}$, Tae Kyoung Kim ${ }^{6}$, Veronica Salvatore', Juergen Karl Willmann ${ }^{7}$, Claude B. Sirlin ${ }^{8}$, Yuko Kono ${ }^{9}$

Affiliations

1 Unit of Internal Medicine, Dpt of Medical and Surgical Sciences, University of Bologna, S.Orsola-Malpighi hospital, Bologna, Italy

2 Radiology and Medicine, Division of Gastroenterology, University of Calgary, Canada

3 Department of radiology, Thomas Jefferson University Hospitals, Philadelphia, United States

4 Clinical Sciences, Imperial College, London, United Kingdom of Great Britain and Northern Ireland

5 Unit of Internal Medicine 2, Caritas-Krankenhaus, Bad Mergentheim, Germany

6 Department of Medical Imaging, University of Toronto, Canada

7 Department of Radiology, Stanford University, Stanford, United States

8 Liver Imaging Grup, Department of Radiology, University of California, San Diego, United States

9 Department of Medicine and Radiology, University of California, San Diego, United States
Key Words

diagnostic radiology, contrast agents, tumor

received 30.9 .2016

accepted 26.12.2016

Bibliography

DOI http://dx.doi.org/10.1055/s-0042-124661

Published online: March 22, 2017 | Ultraschall in Med 2017;

38: 320-324

(c) Georg Thieme Verlag KG Stuttgart · New York

ISSN 0172-4614

Correspondence

Prof. Fabio Piscaglia

Dept of Medical and Surgical Sciences, Div. Internal Medicine via Albertoni 15

40138 Bologna

Italy

Tel.: ++ 39/0 51/2142542

Fax: ++39/0 51/6362725

fabio.piscaglia@unibo.it

\section{Introduction}

The Liver Imaging Reporting and Data System ${ }^{\circledR}$ (LI-RADS ${ }^{\circledR}$ ) was designed and introduced by the American College of Radiology (ACR) to standardize the reporting and data collection of CT and MR imaging for hepatocellular carcinoma (HCC) [http://www.acr.org/ quality-safety/resources/LIRADS]. In 2014, the ACR further identified an international working group of radiologists and hepatologists with expertise in CEUS, including many of the authors of the current article, to establish criteria for CEUS LI-RADS ${ }^{\circledR}$, in collaboration with the LI-RADS ${ }^{\circledR}$ Steering Committee. A key goal was to harmonize the interpretation of CEUS with that of CT and MR and to facilitate future integration of all imaging techniques for HCC diagnosis. The CEUS LI-RADS ${ }^{\circledR}$ system was officially released by the ACR and published online in September 2016 [http://www.acr.org/quality-safety/resources/LIRADS] ( $\bullet$ Fig. 1).

All LI-RADS ${ }^{\circledR}$ comprise an algorithm which categorizes observations from LR-1 ( $\triangleright$ Fig. 2), a definitely benign lesion, through to LR-5, reflecting certainty in the diagnosis of HCC (definitely HCC). LR-M suggests a malignant lesion without specificity for HCC and LR-V suggests macrovascular invasion of a portal or hepatic vein (tumor in vein). Between LR-1 and LR-5 are all other possible observations, including hepatocellular nodules reflecting the process of hepatocarcinogenesis, such as LR-2, a probably 
The American College of Radiology CEUS LI-RADS ${ }^{\circledast}$ Algorithm

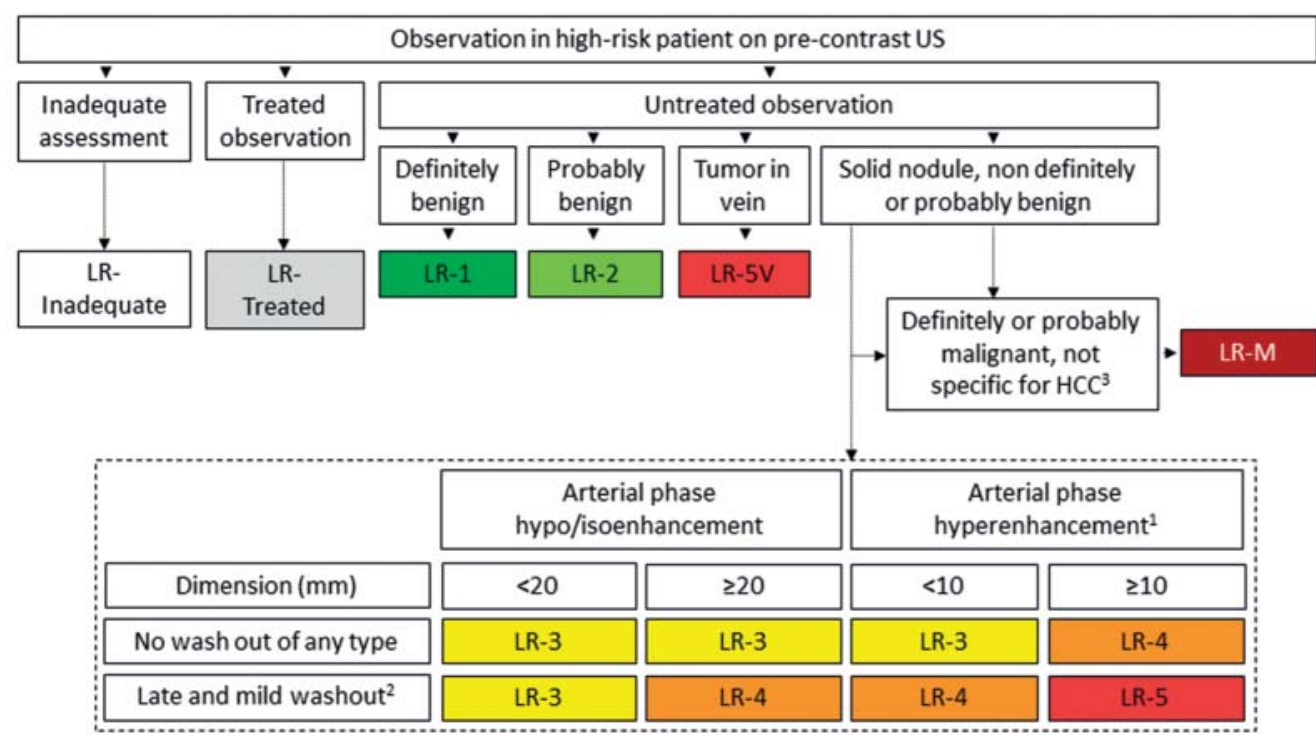

Apply ancillary features and then apply tie-breaking rules to adjust category as appropriate

\begin{tabular}{l}
\hline \multicolumn{1}{|c|}{ LR-1 } \\
\hline - Simple cyst \\
- Classic hemangioma \\
- Definite focal hepatic fat \\
deposition or sparing \\
\hline \multicolumn{1}{|c|}{ LR-2 } \\
\hline - Isoenhancement in all phases \\
- Distinct solid nodule <10mm \\
OR \\
- Not a distinct solid nodule, \\
any dimension \\
- Observation previously LR-3 \\
and stable dimension for 2 \\
years or more
\end{tabular}

\begin{tabular}{|l|}
\hline \multicolumn{1}{|c|}{ LR-5V } \\
\hline - Definite enhancing soft \\
tissue in vein regardless of \\
visualization of parenchymal \\
mass/nodule
\end{tabular}

\begin{tabular}{|l|}
\hline \multicolumn{1}{|c|}{ LR-M } \\
\hline Washout characteristics: \\
- Early onset washout \\
(<60sec) and/or marked \\
(punched out) appearance \\
Arterial phase enhancement \\
- Rim enhancement \\
\hline
\end{tabular}

${ }^{1}$ Arterial phase hyperenhancement: whole or in part, not rim or peripheral discontinuous globular enhancement ${ }^{2}$ Late in onset ( $\geq 60$ seconds) and mild in degree: in whole or in part, with no part showing early or marked washout ${ }^{3}$ Early onset washout ( $<60$ seconds) and/or marked (punched out) appearance and/or arterial phase rim enhancement

- Fig. 1 CEUS LI-RADS Algorithm American College of Radiology (ACR) CEUS LI RADS diagnostic flow-chart, [http://www.acr.org/quality-safety/ resources/LIRADS].
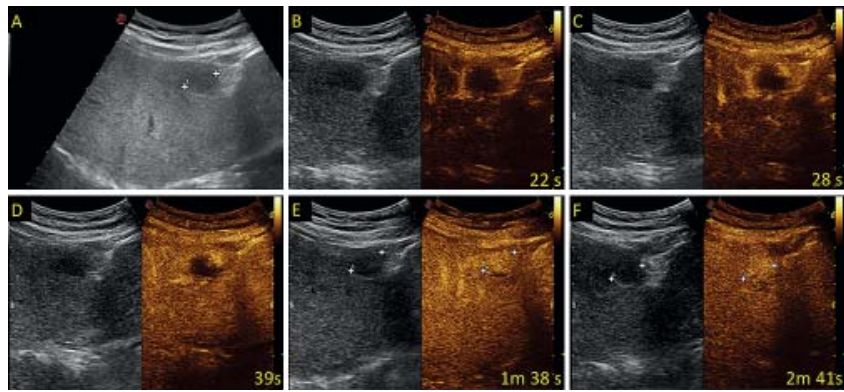

- Fig. 2 LR-1: Definitely benign. 62-year-old male with newly diagnosed alcohol-induced cirrhosis with a focal liver lesion. A, subcostal epigastric transverse B-mode image shows a hypoechoic, $21 \mathrm{~mm}$ subcapsular lesion (calipers) located anteriorly in segment 3. B, C, CEUS shows peripheral globular enhancement during the arterial phase. D, E, $\mathbf{F}$, show progressive centripetal filling in the portal and late phases leading to uniform hyperenhancement in the venous phase, consistent with a classic hemangioma. The lesion is categorized as LR-1.

benign observation ( $\vee$ Fig. 3), LR-3, a observation of intermediate probability for HCC ( Fig. 4, 5), and LR-4 (\Fig. 6, 7), a nodule with high probability for HCC. The categories LR-3 to LR-5 tend to roughly reflect the progression from dysplastic nodules (>4 mm) to mature HCC, paralleled by a derangement of intratumoral vascularization (Matsui O, et al. Abdom Imaging 2011;36:264 - 272; Claudon M et al, Ultraschall in Med 2013;34:11-29).
The CEUS LI-RADS ${ }^{\circledR}$ working group adopted a conservative approach in order to preserve an extremely high (ideally $100 \%$ ) positive predictive value for the diagnosis of HCC in lesions characterised as LR-5 ( $\triangleright$ Fig. 8). Therefore, the diagnosis of HCC with CEUS LI-RADS can be trusted with great confidence, and it virtually eliminates the risk of misdiagnosing cholangiocarcinoma. Stringent criteria are required to achieve such high specificity for HCC, which unavoidably reduces sensitivity. Hence, HCCs may occur in other categories, most commonly well differentiated HCC in categories LR-3 and LR-4 and poorly differentiated HCC in LR-M, where diagnoses are achieved by histology, in keeping with the extant hepatology guidelines (Bruix J, Sherman M. Hepatology 2011;53:1020 1022; Bolondi L et a, Dig Liver Dis 2013; 45: 712 - 723).

To address the problem of diagnosis of non-hepatocellular malignant lesions by CEUS, which often corresponds to mass forming peripheral cholangiocellular carcinoma (de Sio I, United European Gastroenterol J 2014;2:279 - 287; Wildner D, et al. Ultraschall in Med. 2014;35:522 - 7; Yuan, M. X, Ultraschall in Med, 2016; 37: 609-618), CEUS LI-RADS requires meticulous assessment of the timing and degree of contrast washout and of the intranodular contrast distribution in the arterial phase. A rim enhancement distribution in the arterial phase is not typical of HCC and should be categorized under LR-M ( $>$ Fig. 9). Washout that is mild in degree and late in onset (start $\geq 60$ seconds after injection) is consistent with a hepatocellular lesion (Boozari B, et al. Dig Liver Dis. 2011;43:484-90; Wildner D, et al. Ultraschall in Med. 

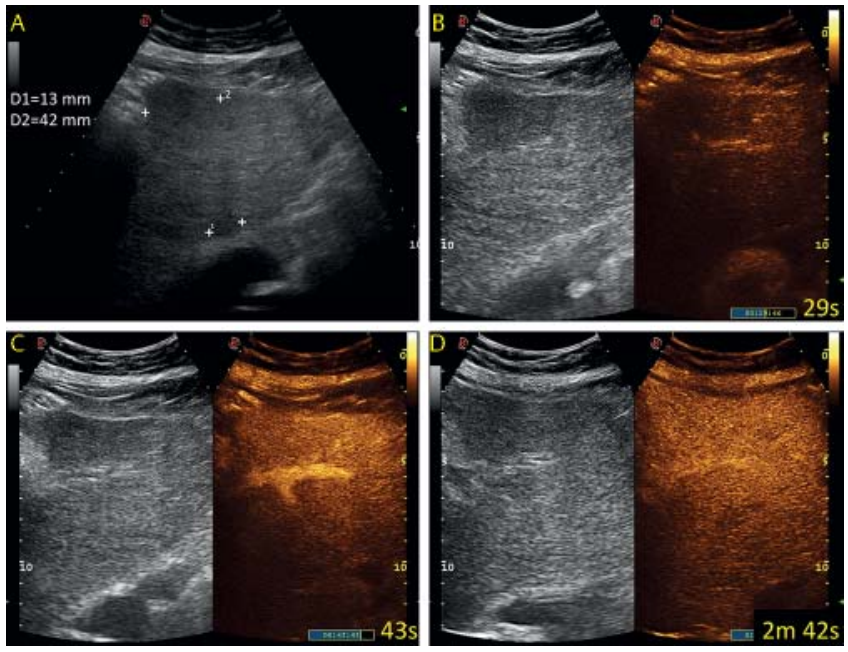

Fig. 3 LR-2: Probably benign. 73-year-old overweight male with alcohol-induced liver cirrhosis displays a component of bright (fatty) echotexture. A, B-mode image reveals a $13 \mathrm{~mm}$ hypoechoic observation (calipers, d1) in the dorsal region of segment 2 and a $42 \mathrm{~mm}$ observation (calipers, d2) subcapsular in segment 3 . Because of the risk of hepatocellular neoplasm in this patient, CEUS was performed. B-D, CEUS shows no evidence of abnormal vascularity in either observation, showing instead isoenhancement equal to the surrounding liver in all phases. This confirms the suspicion of pseudolesions from fatty sparing, to be categorized as LR-2, due to the atypical location of the fatty sparing.
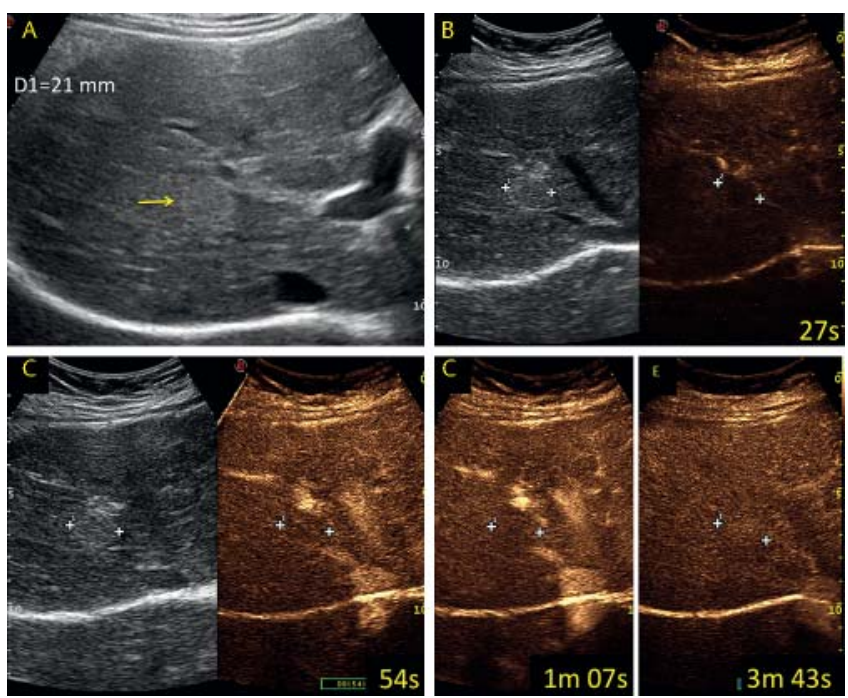

- Fig. 4 LR-3: Intermediate probability for HCC. 48-year-old female patient with autoimmune cirrhosis. A, a conventional B-mode US image shows a subtle hyperechoic $21 \mathrm{~mm}$ nodule (arrow). B, an arterial phase CEUS image shows isoenhancement of the nodule (calipers). C-E are portal venous and late phase images showing persistent isoenhancement with no washout. The nodule is categorized as LR-3.

2015;36:132 -9). Therefore, depending on nodule diameter and arterial features, a lesion with this type of washout can be categorized LR-5 ( $\triangleright$ Fig. 8). By comparison, washout that is marked in degree and/or early in onset (start $<60$ seconds after injection) is non-typical of hepatocellular origin (Yuan MX, et al. Ultraschall in
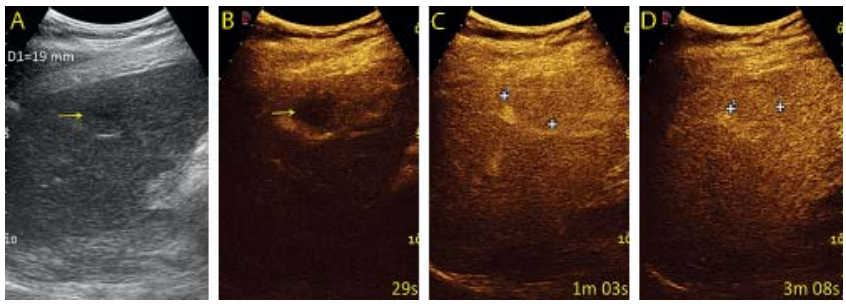

- Fig. 5 LR-3: Intermediate probability for HCC. 68-year-old male with $\mathrm{HCV}$ related liver cirrhosis and prior resection for early stage single HCC three years before. A On follow up surveillance US, a $19 \mathrm{~mm}$ hypoechoic liver nodule is detected (right intercostal scan, segment 6). B, an image in the arterial phase of CEUS shows hypoenhancement. C-D, portal venous and late phase images both show isoenhancement (calipers mark the nodule). The lesion is categorized as LR-3. Biopsy showed a low grade dyplastic nodule. The nodule progressed to LR- 5 pattern after size increase over 18 months).
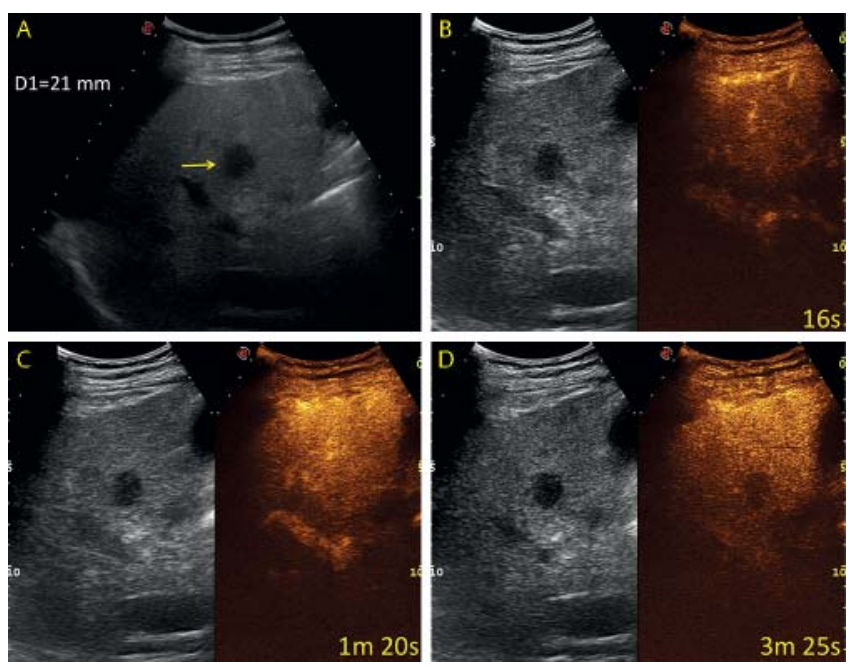

- Fig. 6 LR-4: Probably HCC. 55-year-old diabetic patient with chronic HBV-induced liver fibrosis A, a B-mode image shows a $21 \mathrm{~mm}$ hypoechoic nodule (arrow) in segment 8 . B, an arterial phase CEUS image shows isoenhancement. $\mathbf{C}$, in the portal venous phase the nodule maintains isoenhancement, with no washout. $\mathbf{D}$, late phase image after 3 minutes shows mild and late washout. The lesion is to be categorized as LR-4.

Med. 2016; 37: 609 - 618); regardless of other features, a lesion with this washout pattern is categorized LR-M ( $\vee$ Fig. 10). Although the degree of washout is critical to the interpretation, the judgement is left to the operator, since washout degree is influenced by the amount and type of administered contrast material as well as by the ultrasound machine (which may convey different sensitivity to contrast). Investigators from Erlangen recently proposed a CEUS diagnostic system by modifying the CT MRI LI-RADS criteria to incorporate an assessment of washout timing. The Erlangen system does not address the degree of washout (Schellhaas B, et al. Ultraschall in Med. 2016, 2016; 37: 627 -634), however, thus differing from the official ACR CEUS LIRADS $^{\circledR}$ criteria [http://www.acr.org/quality-safety/resources/LIRADS]. Despite addressing only the timing of washout, the Erlangen system was able to provide $75 \%$ positive predictive value for 

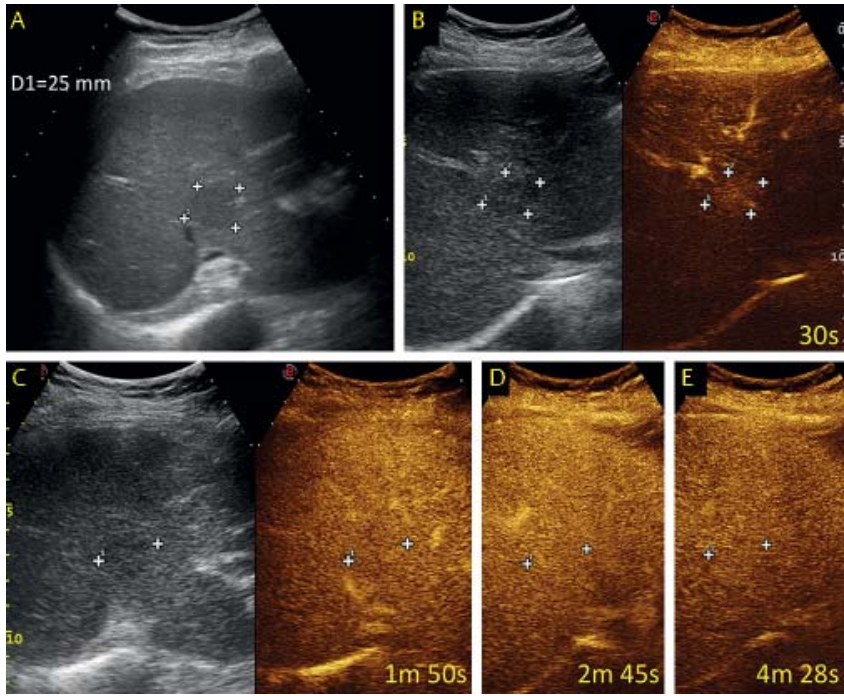

- Fig.7 LR-4: Probably HCC. 73-year-old female with chronic HCVinduced liver cirrhosis. A, B-mode US image shows a $25 \mathrm{~mm}$ nodule (CAUPERS) in segment 4A/8. B, an arterial phase CEUS image shows mild global hyperenhancement. C-E, Sequential images in the portal and late phases show no washout such that the lesion is isoenhancing throughout. Since there is no late phase washout, the lesion is categorized as LR-4. It does not achieve LR- 5 status. The pattern, in association with a identified baseline nodule on US, as here, is associated with a high probability of HCC and most likely of well differentiated grade.
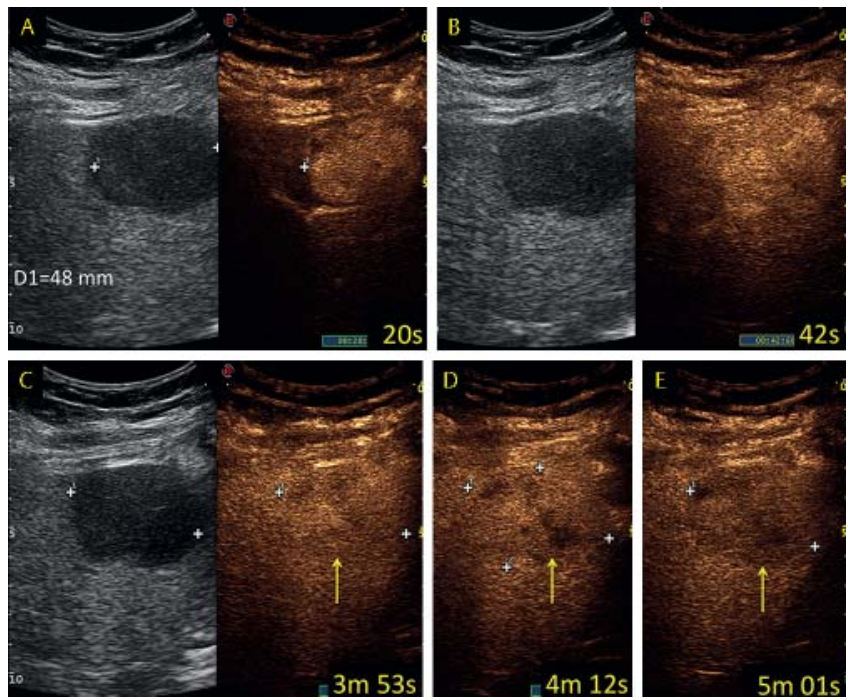

- Fig. 8 LR-5: Definitely HCC. 73-year-old male with alcohol-induced fatty and cirrhotic liver receiving concurrently the diagnosis of a $48 \mathrm{~mm}$ focal liver lesion (nodule calipers) in segment 4 and cirrhosis during an US scan requested because of abnormal liver enzymes. A, the arterial phase of CEUS shows hyperenhancement. B, at the transition between the arterial and portal venous phase the nodule becomes isoenhancing (B). In the late phase, there is a heterogenous, mild and late washout beginning only after 3 minutes. (C-D). 5 minutes after contrast injection the washout is more obvious $\mathbf{E}$. The patterns is categorized as LR-5. Moderately differentiated HCC was confirmed at histology following resection.
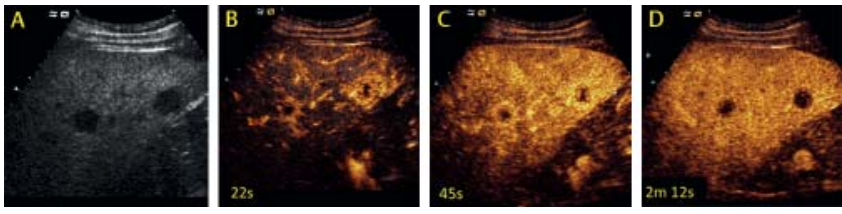

- Fig. 9 LR-M: Malignant lesion, without specificity for HCC 69year-old male patient with suspected liver cirrhosis, splenomegaly, minimal perisplenic ascites and thrombocytopenia. The patient was found to suffer from a large heterogeneous liver mass and distant hypoechoic rounded lesions in the right lobe, visible at B-mode grayscale US (A). CEUS images show rim-like hyperenhancement of the two hypoechoic lesions in the arterial phase (B), still visible at 45 seconds after contrast injection (C) and followed by marked wash out in the venous phase (D). Final biopsy based diagnosis was cholangiocellular carcinoma with intrahepatic metastases.

non-HCC malignancy in a single-center study (Schellhaas B, et al. Ultraschall in Med. 2016, 2016; 37: 627 -634).

Another conservative decision by the CEUS LI-RADS ${ }^{\circledR}$ working group was to require a size threshold of $10 \mathrm{~mm}$ for LR-5 categorization. This was done to maintain consistency with $C T / M R I$ LI-RADS $^{\circledR}$, which does not allow LR-5 categorization for $<10 \mathrm{~mm}$ observations, while recognizing the difficulty of confidently characterizing such tiny nodules by non-invasive imaging. While not allowing $<10 \mathrm{~mm}$ nodules to be categorized LR-5 may cause delayed diagnosis in some small HCCs, there is no evidence that delayed diagnosis of small nodules adversely affects patient outcome as long as the patients are monitored closely.

This pictorial essay seeks to familiarize potential users with the system to facilitate its proper clinical adoption. To accomplish this goal, the display reviews the CEUS LI-RADS ${ }^{\circledR}$ algorithm ( $\triangleright$ Fig. 1) and illustrates the spectrum of CEUS LI-RADS lesions. According to the flow chart, the first step is to assess whether the contrastenhanced ultrasound exam is adequate. Any observations that cannot be assigned a reliable category due to significant problems with contrast injection, lesion depth, liver attenuation, etc., should be reported as LR-inadequate, Treated lesions should be reported as LR-treated ( $\triangleright$ Fig. 11) and are not currently further classified under the LI-RADS ${ }^{\circledR}$ system (this is work in progress from ACR). Presence of enhancing, perfused soft tissue material in any hepatic vessels (hepatic vein or portal vein) corresponds to a tumor in the vein, even in the absence of a clearly detectable parenchymal HCC or LR-M lesion and is characterised as LR-V ( $\triangleright$ Fig. 11). If the soft tissue in the vein does not fit the LR-V category it could be classified as bland non tumor related thrombus (Claudon M et al, Ultraschall in Med 2013;34:11 - 29).

Subsequently the CEUS pattern of any identified focal observation is analyzed, recognizing that on US, virtually all observations are true nodules (apart from focal fat infiltration or sparing), unlike CT and MR. A pattern corresponding to definitely benign entities such as hemangiomas and simple cysts is categorized as LR-1. Focal fat deposition and focal fat sparing, with classic enhancement characteristics, can also be classified as definitely benign, LR-1. Next, it is judged whether the CEUS pattern corresponds to a definitively malignant lesion, but of uncertain cellular origin which is to be categorized LR-M. The cellular characterization of LR-M lesions requires histological confirmation: it often corresponds to cholangio- 

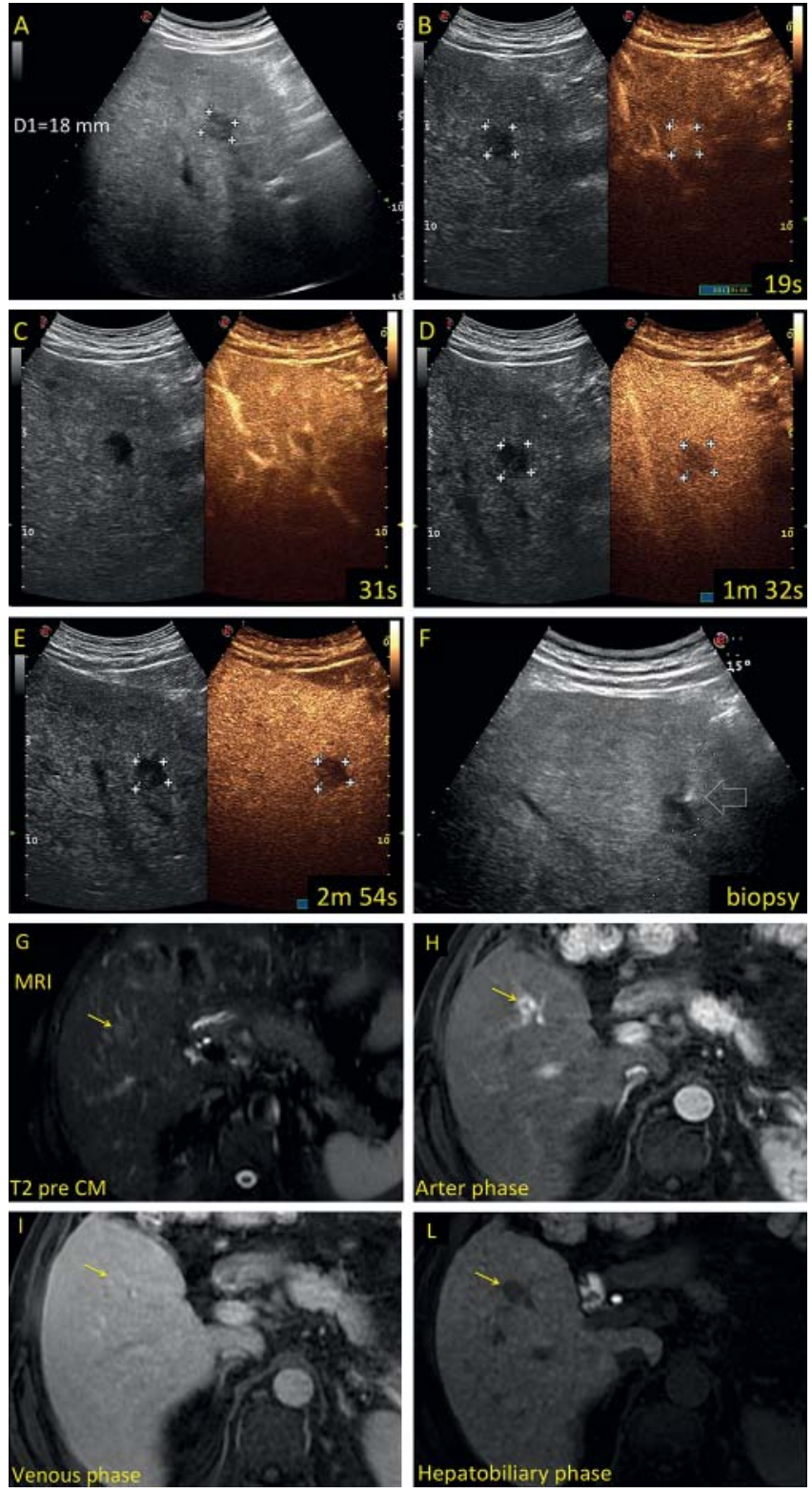

- Fig. 10 Malignant lesion, without specificity for HCC. 59-year-old male with alcohol and metabolic syndrome induced liver cirrhosis showed a new liver nodule during surveillance. A, B-mode US image shows an $18 \mathrm{~mm}$ hypoechoic nodule in segment 4 (calipers). B, CEUS image shows mild homogenous hyperenhancement (calipers) on arterial phase. C, By as early as 31 seconds, there is partial washout, which becomes more evident with time (D) and marked after 2 minutes (calipers, panel E). Due to early and marked wash out, this is classified as LR-M. F, US guided biopsy showed cholangiocarcinoma (empty arrow indicates the tip of the needle). Indeterminate MR scan on former patient. G, the same nodule is identified on the precontrast MR scan. $\mathbf{H}$, MR arterial phase with EOB gadolinium shows a thick rim hyperenhancement (almost global hyperenhancement). I, in the portal venous phase, there is no washout. L, on the hepatobiliary phase, there is an enhancement defect.

carcinoma, but some lesions categorized LR-M are poorly differentiated or otherwise atypical HCC or quite rarely other rare entities. If a sonographically distinct nodule does not fit any of the previously mentioned categories, then it is likely to be of hepatocellular type
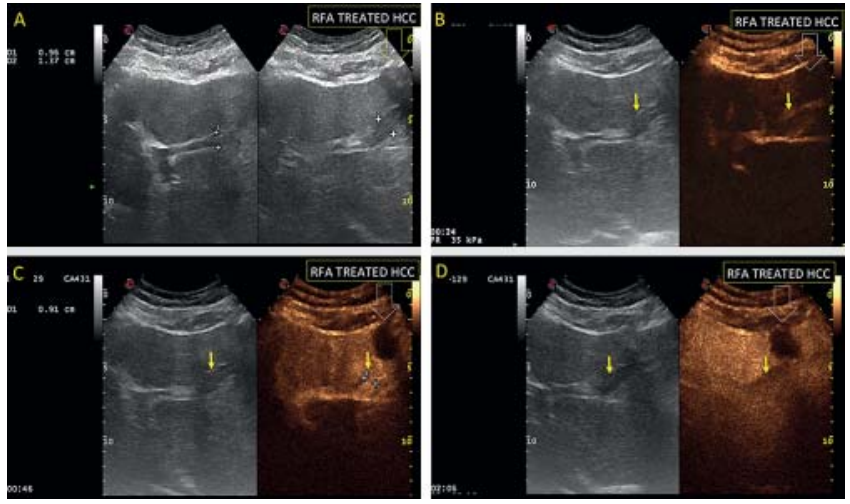

- Fig. 11 LR-V: Tumor in vein and LR-treated: treated observation. 52-year-old HIV positive male with HBV and HCV cirrhosis, on the waiting list for liver transplantation for a single HCC treated with Radiofrequency Ablation (RFA). A At 9 months after RFA, ultrasound shows the appearance of soft echogenic tissue in the segmental portal branch (calipers) afferent to the region of the treated tumor, which is barely recognizable as a slightly hypoechoic nodule (empty arrow). The occluded portal branch appears in direct continuity with the tumor and the portal vein walls are focally disrupted and not well recognizable. The portal branch shows a mass forming aspect since it appears larger downstream. The overall pattern raises a strong suspicion of tumor in the vein. CEUS shows enhancement of the soft tissue in the portal vein both in the arterial (faint hyperenhancement, B) and portal (C) phases and, washout in the late phase (D). The pattern is to be classified as LR-V, consistent with an advanced stage HCC, leading to removal from the transplant waiting list. The treated tumor appears free of enhancement in all phases (B, C, D), consistent with a treated nodule (LR-Treated), not to be confused with LR-M, based also on the clinical history.

and is hence categorized according to its enhancement pattern and size, with increasing probability of being an HCC, from LR-2 (which corresponds most likely to a benign lesion), to LR-3 (intermediate probability of HCC, LR-4 high probability of HCC and LR-5 definitively HCC). Please note that even for LR-2 to LR-3 there is no guarantee that a lesion is not an HCC, even though the probability of HCC is low (most likely a benign lesion) or intermediate, where for LR-4 the probability of HCC is high.

\section{Conflict of Interest}

Fabio Piscaglia: Speaker fees: Bayer, Bracco; Advisory board: Bayer; Research contract: Esaote. / Stephanie R. Wilson: Research support: Siemens, Philips; Advisory role: Lantheus / Andrej Lyshchik: Industry grant and Research support: GE, Siemens, Toshiba; Advisory Board: BRACCO; Speaker Panel: SonoScape / David Cosgrove: Speaker and MAB: Bracco Spa, Toshiba, Bk Medical, Clearstream / Christoph F. Dietrich: Advisory board: Hitachi, Mindray; Speaker honorarium: Hitachi, Supersonic, Siemens, Mindray, GE, Bracco, Pentax / Juergen K. Willmann: Research grant: Siemens, GE, Philips, Bracco; Consulting fees: Bracco, Triple Ring Technologies; Advisory board: Lantheus, Bracco, SonoVol / Claude B. Sirlin: Industry grant support: Siemens, GE, Gerber; Consulting and service agreements: Bracco / Yuko Kono: Grant and research support: Toshiba, GE, Philips; Advisory board and speaker fees: Bayer, Wako 